\title{
M giants foundin the First Byurakan Spectral Sky data base. V. Gaia DR2 data.
}

\author{
Gigoyan K.S.* \\ V .A. Ambartsumian Byurakan Astrophysical Observatory, Armenia
}

\begin{abstract}
In this paper we study bright M-type giants found in the First Byurakan Survey (FBS) lowresolution (lr) spectroscopic data base. Phase dependence light-curves from large sky area variability data bases such as Catalina Sky Survey (CSS) and All-Sky Automated Survey for Supernovae (ASAS-SN), and the second Gaia data release data (Gaia DR2) high-quality photometric data are analyzed to estimate some important physical parameters for $1096 \mathrm{M}$-type giants found at high Galactic latitudes. Their Gaia DR2 broad-band G magnitudes are in the range $8.0<\mathrm{G}<16.0$ mag. Gaia DR2 radial velocities (RV) are available for 134 and luminosities for 158 stars out of 1096. The Gaia DR2 color-absolute magnitude diagram (CaMD), their Galactic distribution, also some other diagrams based on Gaia DR2 photometric data are presented. Absolute magnitudes cover the range $+1.0 \leq \mathrm{M}(\mathrm{G}) \leq-5.4 \mathrm{mag}$. They follow to the behaviors and occupy the same regions on the color-magnitude diagrams studied in many papers by different researches for long period variables (LPVs). Particularly, we consider the locations of the FBS giants on the new diagrams, using multi-band approaches in combination with Wesenheit functions, obtained recently by Lebzelter and colleagues (2018) for the oxygen-rich (O-rich) and carbon-rich (C-rich) LPVs in the Large Magellanic Cloud. Period-Luminosity (P-L) diagram was presented for 112 M Mira-variables. The upper limit of the initial stellar masses can be estimate near $5 \mathrm{M}_{\odot}$ for $\mathrm{M}$ giants according to the new diagnostic tools. The kinematic properties, space distribution also more interesting cases among the sample were considered.
\end{abstract}

Keywords: Late-type stars: Astronomical data bases-Surveys.

\section{Introduction}

The First Byurakan Survey (FBS), known also as the Markarian survey, was the first systematic objective-prism survey of the extragalactic sky. This survey was conducted by B. E. Markarian and collaborators from 1965 to 1980. The photographic plates were obtained at the Byurakan Astrophysical Observatory (BAO) using the 1-m Schmidt telescope, equipped with a $1.5^{\circ}$ prism, giving a reciprocal dispersion of $1800 \AA / \mathrm{mm}$ near $\mathrm{H}_{\gamma}$ throughout a useful field of $4^{\circ} \times 4^{\circ}$. FBS is large -area low-resolution (lr) spectral survey, covering a total of $17.000 \mathrm{deg}^{2}$. It is segmented into 28 parallel zones on all the Northern sky and part of the Southern sky at high Galactic latitudes with $\delta>-15^{\circ}$ and $|\mathrm{b}|>15^{\circ}$. The limiting magnitude is 17.5-18.0 mag. in the photographic bandpass. Various Kodak emulsions were used during the observations (IIF, IIAF, IIaF, and $103 \mathrm{aF}$ ), providing a spectral range of 3400-6900 $\AA$ (with a $70 \AA$ - wide sensitivity gap at $5300 \AA$ ) ), and spectral resolution of $\mathrm{R}=96$ near $\mathrm{H}_{\gamma}$. The FBS was originally conducted to search for galaxies with an ultraviolet excess (UVX; Markarian, 1967). In total, 1515 UVX galaxies (Markarian galaxies) had been discovered, including many AGN, Starburst and Seyfert galaxies (Markarian et al., 1989). The discovery of UVX galaxies by Markarian and collaborators was the first and most important work based on the FBS spectroscopic plates. Several other interesting projects based on the FBS were started in 1987 (known as a second part of the FBS, Abrahamian \& Mickaelian (1996)), which resulted in the discovery of new bright quasi-stellar objects (QSOs), Seyferts, white dwarfs (WDs), subdwarfs (sds), planetary nebula nuclei (PNNs), cataclysmic variables (CVs), and other interesting objects (Mickaelian, 2008). 


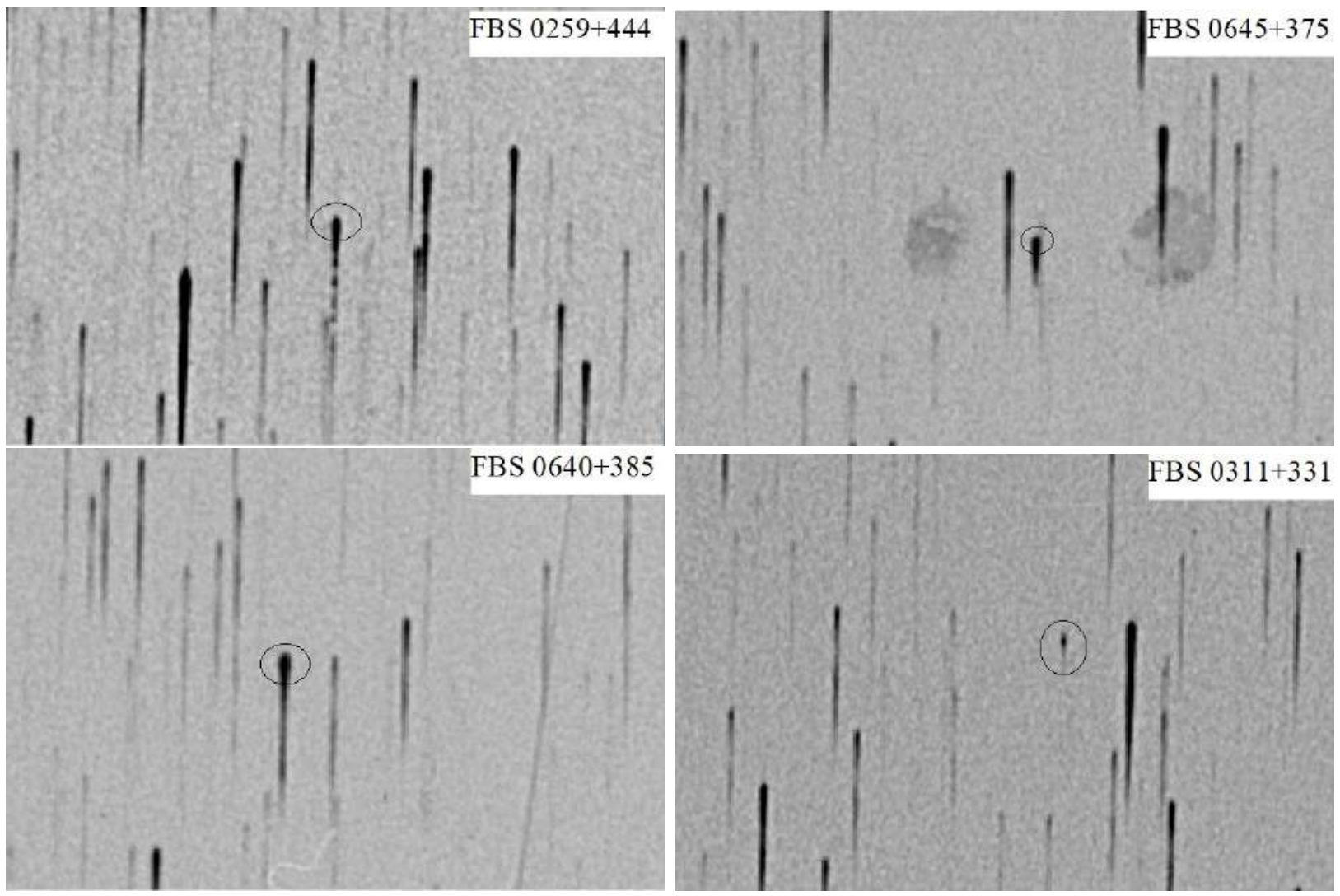

Figure 1. FBS lr spectral shapes for M and C stars

The second part of the FBS also included the selection, cataloguing and study of faint late-type stars (LTSs) at high Galactic latitudes. The large spectral range of the FBS is also suited to identify M-type and carbon (C)-stars. C stars can be identified through the presence of the Swan bands of the $\mathrm{C}_{2}$ molecule $(4383,4737,5165$, and $5636 \AA$ ), M-type spectra can easily be separated thanks to titanium oxide ( $\mathrm{TiO}$ ) molecular absorption bands (4584, 4762, 4954, 5167, 5448, 5862, 6159, and $6700 \AA$ A). Since 2007 all FBS lr plates have been digitized, resulting in the creation of the Digitized First Byurakan Survey (DFBS) database (Mickaelian et al., 2007), for details see the web sites at http://www.ia2-byurakan.oats.inaf.it/ and http://www.aras.am/Dfbs/dfbs.html/ ). Figure 1 presents the FBS lr spectral shapes for early and late subclasses of the $\mathrm{C}$ and $\mathrm{M}$ stars. All DFBS plates were analyzed with the help of standard image analysis softwares. The sec- ond version of the "Revised And Updated Catalogue of the First Byurakan Survey of Late-Type Stars", containing data for 1471 LTSs (130 C-type stars, $235 \mathrm{M}$ dwarfs, and $1096 \mathrm{M}$-type giants, was generated (Gigoyan et al. (2019), SIMBAD Vizier catalogue J/MNRAS/489/2030/catv2).

\section{Optical Spectroscopy}

For FBS LTSs, medium-resolution CCD spectra were obtained at different epochs with the BAO (Armenia) 2.6-m telescope ( UAGS, ByuFOSC2 and SCORPIO spectrographs), moderate - and highresolution CCD spectroscopy obtained with the Observatory de Haute-Provence (OHP, France) 1.93$\mathrm{m}$ telescope (CARELEC spectrograph). For some FBS LTSs medium-resolution CCD spectra also were obtained with the Cima-Ekar 1.83-m telescope of the Padova Astronomical Observatory (Italy) equipped with the Asiago Faint Objects Spectrometer and Camera (AFOSC), and with the 1.52m Cassini telescope of the Bologna Astronomical Observatory at Loiano (Italy) equipped with the Bologna Faint Objects Spectrometer and Camera (BFOSC; Gigoyan et al., 2019).

Figure 2 presents the 2.6-m BAO telescope moderate-resolution CCD spectra in the range 4000$7250 \AA$ for some amount FBS LTSs.

*kgigoyan@bao.sci.am 

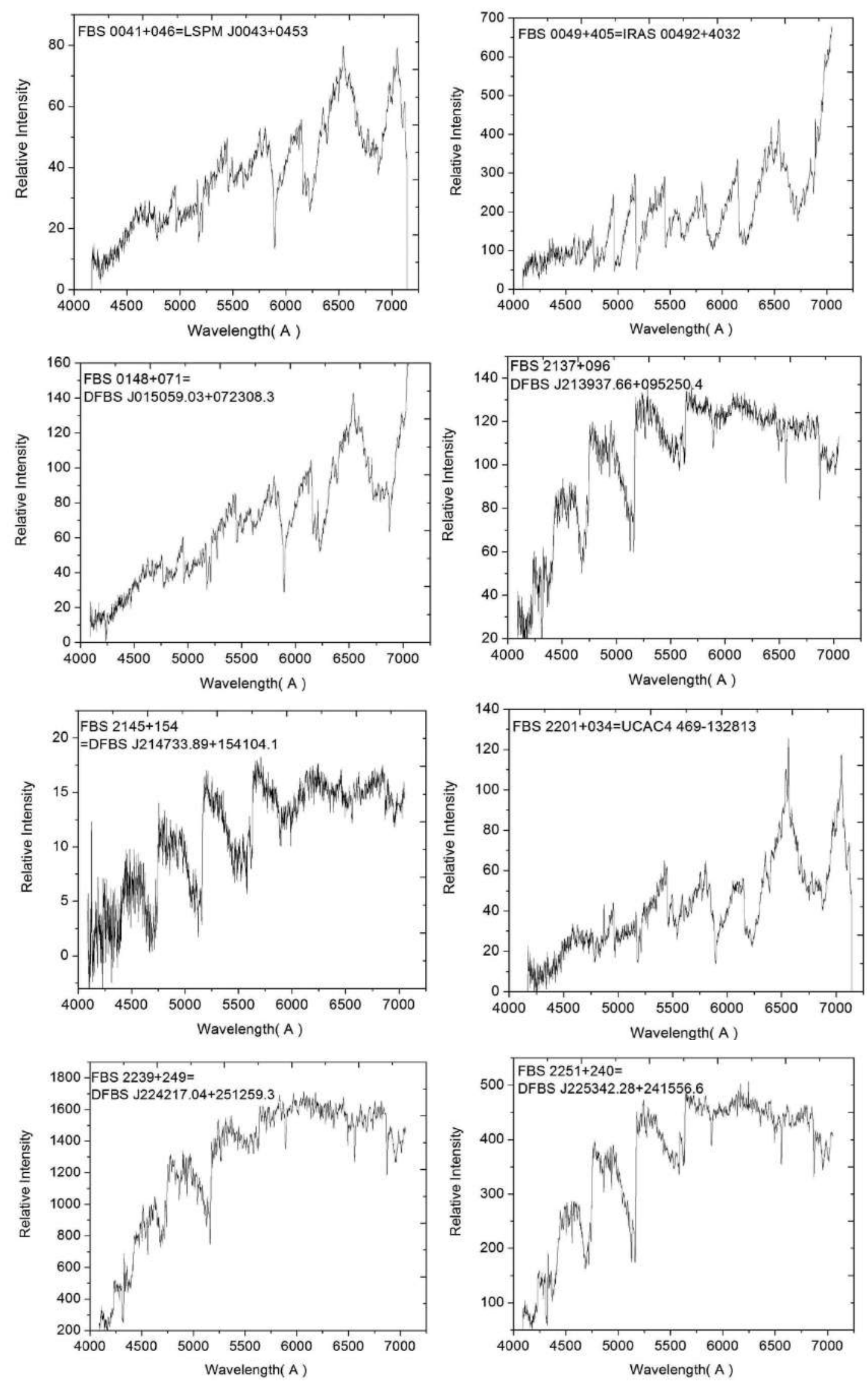

Figure 2. Figure 2. 2.6-m BAO telescope moderate-resolution CCD spectra for some FBS LTSs.

Moderate-resolution CCD spectra for more than 300 FBS LTS were sequred by LAMOST (Large Sky Area Multi-Object Fiber Spectroscopic Telescope) observations (LAMOST DR5; Luo et al., 2019), 
Table 1. Gaia DR2 data for a sample of the FBS M giants.

\begin{tabular}{lcccccl}
\hline \hline $\begin{array}{l}\text { FBS } \\
\text { number }\end{array}$ & Var type & $\begin{array}{c}\text { G mag } \\
(\mathrm{mag})\end{array}$ & $\begin{array}{c}\text { BP-RP color } \\
(\mathrm{mag})\end{array}$ & $\begin{array}{c}\mathrm{T}_{\text {eff }} \\
(\mathrm{K})\end{array}$ & $\begin{array}{c}\text { Luminosity } \\
(\text { solar units })\end{array}$ & Other Association \\
\hline $0001+340$ & L & 10.31 & 3.38 & 3304 & 340.28 & \\
$0212+858$ & SR & 11.83 & 2.44 & 3615 & 999.79 & \\
$0519+021$ & SR & 11.21 & 3.31 & 3306 & 139.47 & \\
$1014+819$ & SR & 10.15 & 2.82 & 3529 & 1357.73 & IRAS 10147+8159 \\
$1201+850$ & L & 10.65 & 3.03 & 3297 & 1100.97 & IRAS F12019+8503 \\
$1306+385$ & SR & 10.06 & 3.30 & 3306 & 2024.77 & NSVS 5041274 \\
$1454-069$ & SR & 9.87 & 3.10 & 3302 & 388.26 & IRAS 14544-0657 \\
$1737+428$ & SR & 11.83 & 3.44 & 3670 & 319.70 & ROTSE J173832.72+425112.8 \\
$1757+194$ & Mira & 8.07 & 2.92 & 3304 & 927.80 & IRAS 17575+1929 \\
$2147+023$ & SR & 9.19 & 4.10 & 3326 & 761.05 & IRAS 21465+0220 \\
\hline
\end{tabular}

spectra available on-line at http://dr5.lamost.org/search/. The O-rich nature could be confirmed for the big part of the FBS M -giants.

\section{Variability.}

To determine the variability of the FBS M type giants, we exploit data from two primary sources, namely the Catalina Sky Survey (CSS, second public data release CSDR2, accessed via http:// nesssi.cacr.caltech.edu/DataRelease/ (Drake et al., 2014, 2017) and the All-Sky Automated Survey for Supernovae ASAS-SN (accessed via https://asas-sn.usu.edu/variables ). ASAS-SN is the first ground-based survey to monitor the entire visible sky to a depth of $\mathrm{V} \leq 17.0$ mag on a regular basis. As a consequence, ASAS-SN was used as the primary source for attributing variability types, periods, and amplitudes to the FBS M giants. Our final sample consist of 690 Semi-Regular (SR)-type, 294 L-type and 112 Mira-type variables.

Figure 3 presents phased ASAS-SN light curves for three FBS M-giants.

\section{Gaia DR2 Data}

With the advent of Gaia mission (Gaia Collaboration; Prusti et al., 2016) a new era in the astronomical research has started. The Gaia DR2 database containing astrometry, three-band photometry, radial velocities, effective temperatures, and information on astrophysical parameter and variability for approximately 1.7 billion sources brighter than $G=21.0$ magnitude (Brown et al., 2018). This database opens a new area for investigations based on the Hertzsprung-Russell diagram (HRD).

All FBS M giants were cross-matched with the Gaia DR2 catalogue sources. The cross-match was carried out using a 5 arcsec aperture around the position of each of our sample stars. They are relatively bright, so that $\mathrm{G}$ - band brightnesses were in the range $8.0 \mathrm{mag}<\mathrm{G}<16.0 \mathrm{mag}$, effective temperatures lay between $3200 \mathrm{~K}<\mathrm{T}_{\text {eff }}<4300 \mathrm{~K}$.

\subsection{Colors and Luminosities}

The radii and luminosities (in Solar units) of $158 \mathrm{M}$ giants of our sample (out of 1096) can be deduced from the Gaia DR2 data base. Luminosities of our target stars range between $\mathrm{L}=28.039 \mathrm{~L} \odot(\mathrm{FBS}$ 0255+193 = LAMOST J025756.28+193228.5, M1 star) and L = 2024.777L $\odot$ (FBS 1306+385=LAMOST J130829.62+381801.4=IRAS 13061+3834, M6 subtype SR-variable). We computed the absolute V-band magnitudes for these objects, adopting that $\mathrm{M}_{V}=+4.81 \mathrm{mag}$ for the Sun (see Table 3 in Andrae et al. 2018, for more details), resulting in an absolute V-magnitude range between $\mathrm{M}_{V}=+1.1$ and $\mathrm{M}_{V}=-3.5$. A representative sample table with Gaia DR2 data is given as Table 1. 


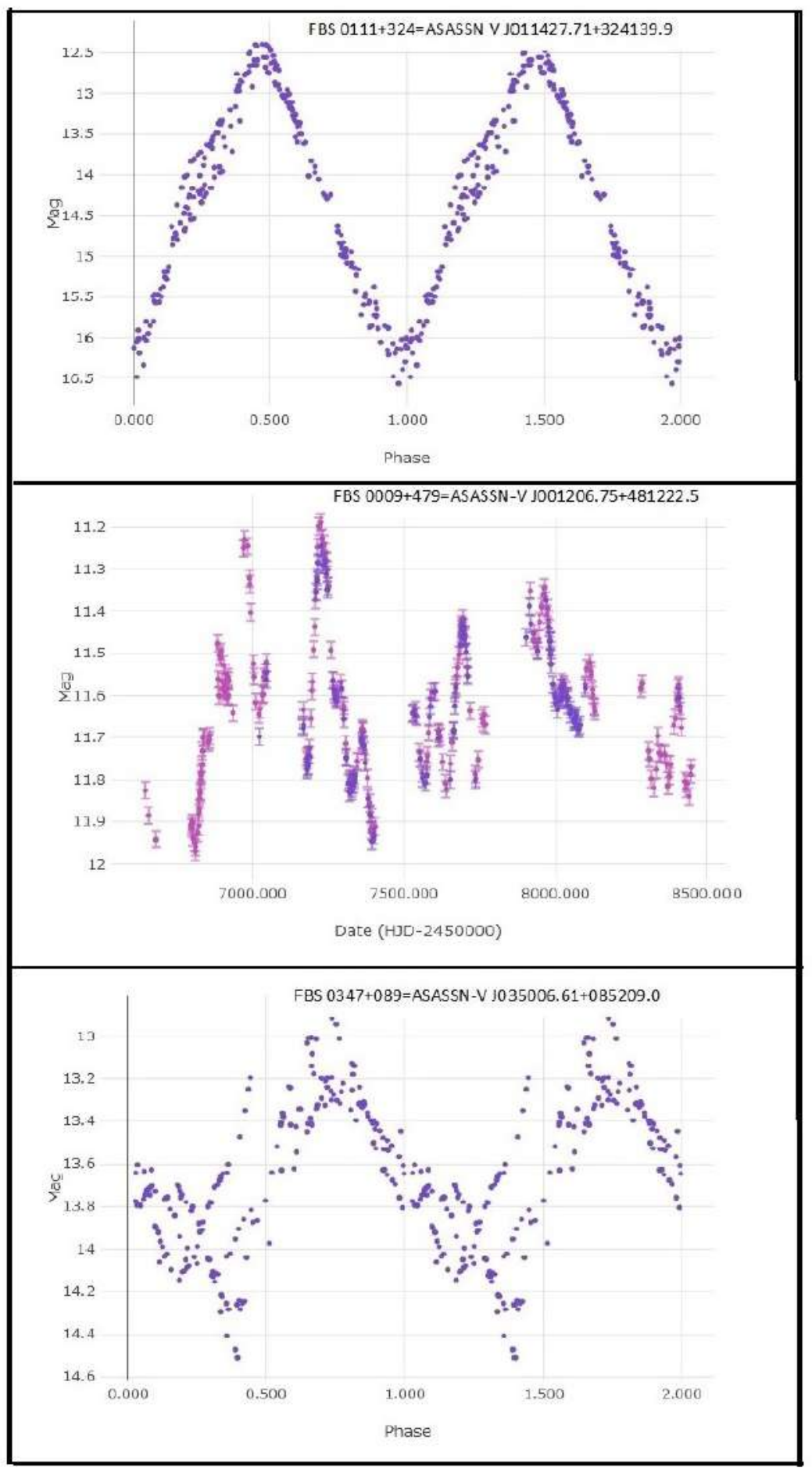

Figure 3. ASAS-SN Phased light curves for FBS 0111+324, FBS 0009+479, and FBS 0347+089, classified consequently as a Mira, SR, and L -type variables.

Figure 4 present the Gaia DR2 color-absolute magnitude (CaMD) diagram for 158 FBS M giants, with available luminosity value in Gaia DR2 data base.

Figure 4. Gaia color-absolute magnitude (MV vs. BP-RP) diagram of FBS M giants with available luminosity data in Gaia DR2 data base. Symbols are: blur circle - SR variables, green square - L variables, and red triangle - Mira - type variables.

We follow the paper by Lebzelter et al. (2018). We use also the distance information derived from Gaia DR2 by Bailer-Jones et al. (2018) (Gaia Collaboration, SIMBAD CDS VizieR catalogue I/347/gaia2dis) to plot the CaMD for FBS M giants. 


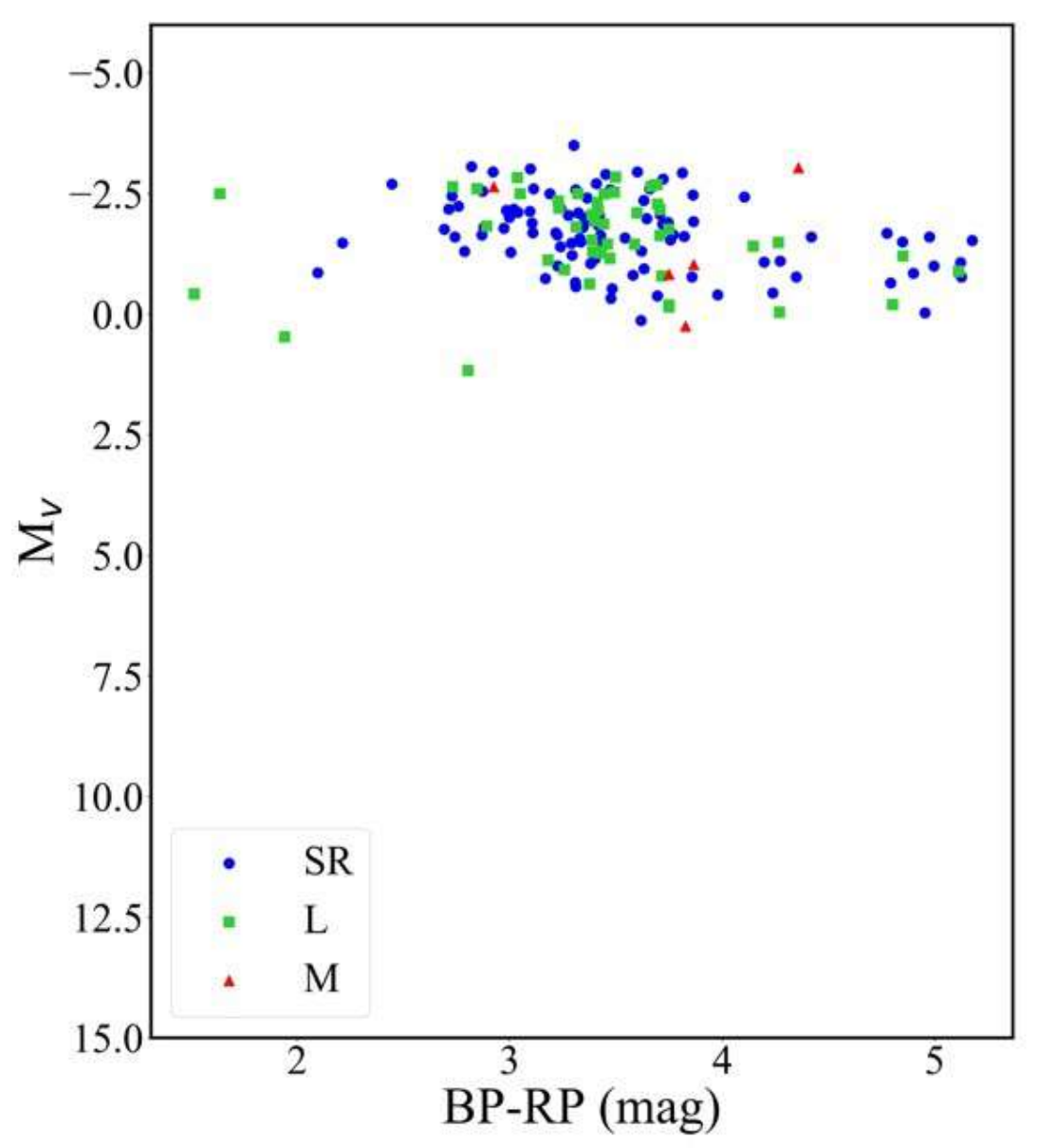

Figure 4. Gaia color-absolute magnitude $\left(\mathrm{M}_{V}\right.$ vs. BP-RP) diagram of FBS M giants with available luminosity data in Gaia DR2 data base. Symbols are: blur circle - SR variables, green square - L variables, and red triangle - Mira - type variables.

\section{2MASS Colors}

Figure 5 presents the 2MASS J- H versus $\mathrm{H}-$ Ks color - color diagram for FBS M stars. In the color - color diagram, objects having $\mathrm{J}-\mathrm{H}>0.8$ and $\mathrm{H}-\mathrm{Ks}>0.2$ are usually giants or asymptotic giant branch (AGB) stars (Bessell \& Brett, 1988).

\section{Discussion And Conclusion.}

We explored the sample of 1096 relatively bright, spectroscopically confirmed M -giants. For this study, we cross-correlated our sample with the data bases from Gaia DR2, the Catalina Sky Survey (CSS), 2MASS, and All-Sky Automated Survey for Supernovae (ASAS-SN). Their Gaia DR2 broadband $\mathrm{G}$ magnitudes are in the range $8.0<\mathrm{G}<16.0 \mathrm{mag}$. Gaia DR2 radial velocities (RV) are available for 134 and luminosities for 158 stars out of 1096.

\section{Acknowledgements}

This research was made possible through the use of the ASAS-SN and Catalina Sky Survey variability data bases. We used the LAMOST telescope spectra. The LAMOST is a National Major Scientific project build by the Chinese Academy of Sciences. This work has made use of data from the European Space Agency (ESA) mission Gaia (https://cosmos.esa.int/gaia). 


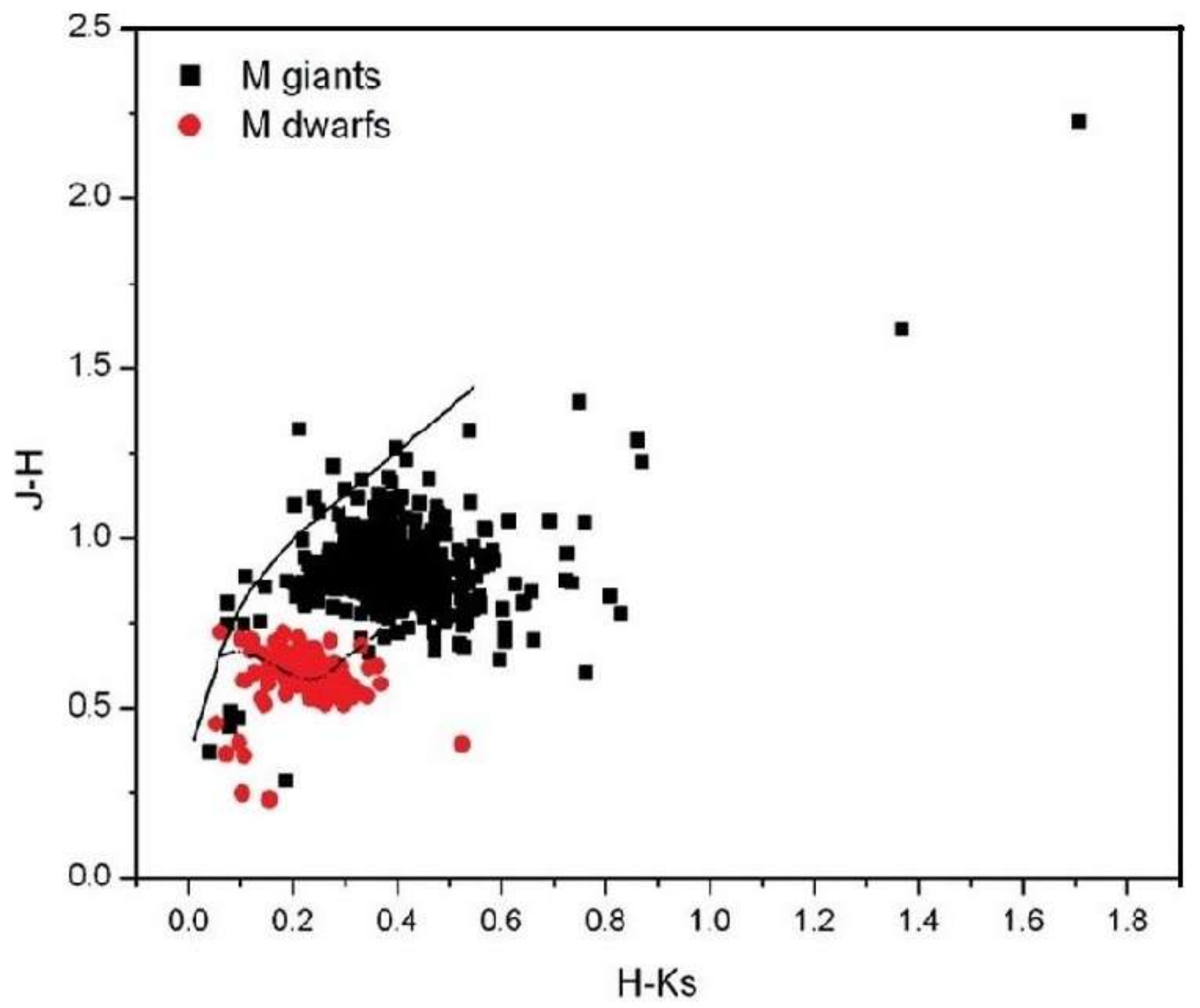

Figure 5. The 2MASS J-H versus H-Ks color-color diagram for FBS M giants. We present also on this diagram a some amount $\mathrm{M}$ dwarfs also for comparison. The solid line shows the $\mathrm{M}$-giant equence and the dashed line with dots denotes the M-dwarf sequence (for more details, see Bessell \& Brett (1988)

\section{References}

Abrahamian H. V., Mickaelian A. M., 1996, Astrophysics, 39, 315

Bailer-Jones C. A. L., Rybizki J., Andrae R., Fouesneau M., 2018, A\&A, 616, A37

Bessell M. S., Brett J. M., 1988, PASP, 100, 1134

Brown A. G. A., et al., 2018, Astron. Astrophys. , 616, A1

Drake A. J., et al., 2014, Astrophys. J. Suppl. Ser. , 213, 9

Drake A. J., et al., 2017, Mon. Not. R. Astron. Soc. , 469, 3688

Gigoyan K. S., Mickaelian A. M., 2012, MNRAS, 419, 3346

Gigoyan K. S., Russeil D., Mickaelian A. M., Sarkissian A., Avtandilyan M. G., 2012, A\&A, 544, A95

Gigoyan K. S., Mickaelian A. M., Kostandyan G. R., 2019, MNRAS, 489, 2030

Lebzelter T., Mowlavi N., Marigo P., Pastorelli G., Trabucchi M., Wood P. R., Lecoeur-Taïbi I., 2018, Astron. Astrophys. , 616, L13

Luo A. L., Zhao Y. H., Zhao G., et al. 2019, VizieR Online Data Catalog, p. V/164

Markarian B. E., 1967, Astrofizika, 3, 55 
Markarian B. E., Lipovetsky V. A., Stepanian J. A., Erastova L. K., Shapovalova A. I., 1989, Soobshcheniya Spetsial'noj Astrofizicheskoj Observatorii, 62, 5

Mickaelian A. M., 2008, AJ, 136, 946

Mickaelian A. M., et al., 2007, Astron. Astrophys. , 464, 1177

Prusti T., et al., 2016, Astron. Astrophys. , 595, A1

Shappee B. J., Prieto J. L., Grupe D., Kochanek C. S., Stanek K. Z.and Walker Z., Yoon Y., 2014, Astrophys. J. , 788, 48 